\title{
AN ITERATIVE GROUPWISE MULTIUSER DETECTOR WITH SOFT OUTPUT
}

\author{
Brad W. Zarikoff ${ }^{1}$, James K. Cavers ${ }^{2}$ \\ ${ }^{1}$ Simon Fraser University, 8888 University Drive, V5A 1S6, Burnaby, BC, Canada, bwzariko@ssu.ca \\ ${ }^{2}$ Simon Fraser University, 8888 University Drive, v5A 1S6, Burnaby, BC, Canada, cavers@sfu.ca
}

\begin{abstract}
We consider a multiuser system in which the interfering users have the same pulse shape and are received in a synchronous, flat fading channel at an antenna array. This paper introduces a new iterative group-wise multiuser detection technique (IMUD) that gives a tradeoff of complexity and performance between joint maximum likelihood (JML) detection and zero forcing (ZF) MUD. It is shown that in the overloaded case the new IMUD algorithm significantly outperforms MMSE V-BLAST. Even with an equal number of users and receive antennas, IMUD gives better performance within two iterations. An additional feature of IMUD is that it inherently produces soft-output decisions.
\end{abstract}

Keywords - Group detection, iterative methods, MAP estimation, MIMO systems, multiuser detection.

\section{INTRODUCTION}

We address multiuser detection for the uplink. The base station antennas experience independent channel gains (a diversity array). Each of the mobile users has a single transmit antenna. All transmit on a flat fading Rayleigh channel. We employ this system model because it is simple and it enables us to examine the essential multiuser problem.

Many techniques for multiuser detection (MUD) exist. The optimum technique is joint maximum likelihood (JML) detection [1]. However, complexity issues force engineers to look elsewhere for a detection scheme, since the number of computations increases exponentially with the number of users in the system.

Linear methods, like decorrelation (zero-forcing, ZF) and minimum-mean square error (MMSE, [6]) apply a filter to suppress mutual interference prior to single-user detections. They are simple, but have much poorer performance than JML, since suppression sacrifices both SNR and diversity.

V-BLAST [5], another suboptimal method, makes a sequence of single-user decisions, with a ZF or MMSE filter to suppress undetected users. Previously detected users are removed by subtraction, a hard cancellation. The order in which users are detected depends on the channel state, unlike the previous single user techniques, and it is the key to its excellent performance.

The group technique proposed by Varanasi [2] occupies a level between JML and ZF. The group detector (GD) separates the users into groups, finds a ZF filter that will effectively null all groups except the current decision group, and then performs a JML decision on all users in a group.

This paper explores a method to extend the group detector in [2] using an iterative process. The new technique, iterative MUD (IMUD), effectively combines the group detection techniques of [2] and the ordering of [5] and introduces a novel use of soft-cancellation and iterative detection. It makes use of the extrinsic information provided when the user groups are re-ordered during the iterative process. Unlike most iterative techniques (turbo codes, for example), the iterations are taken over the groups, not time. It is shown to outperform both the ZF and MMSE group detection techniques ([3],[4]) with only a small complexity boost. A significant performance gain over the MMSE VBLAST is seen in the overloaded case.

\section{SYSTEM MODEL}

We consider the most basic of multi-user interference problems, addressed by Varanasi [2], Golden et al. [5] and $\mathrm{Ng}$ and Sousa [4]: $N$ symbol-synchronous users with identical pulse shapes and single antennas, transmitting through flat fading channels, and received at an $M$ element antenna array with the same SNR at each antenna. Variations such as asynchronous transmission, signature pulse shapes, or delay spread would enhance performance, since they add measurement dimensionality. However, we chose to deal with the simplest system to uncover the fundamental operation and performance of this technique.

In such conditions, the matched filter outputs at the receiver can be represented by the length- $M$ vector

$$
\mathbf{y}=\mathbf{H b}+\mathbf{n}
$$

where $\mathbf{H}$ is the $M \times N$ matrix of channel gains, $\mathbf{b}$ is the length- $N$ vector of user data and $\mathbf{n}$ is the length- $M$ vector of spatially white Gaussian noise. The elements of $\mathbf{H}$ are independent, complex Gaussian variates with zero mean (hence Rayleigh fading) and variance $1 / 2$ in both the real and imaginary components. For simplicity, all users will use the BPSK constellation \pm 1 , although this is not essential. The elements of $\mathbf{n}$ have zero mean and variance $1 / 2 \Gamma$ in both the real and imaginary components, where $\Gamma$ is the SNR per symbol. 


\section{ITERATIVE MULTIUSER DETECTION}

Iterative multiuser detection (IMUD) combines four component techniques: groupwise detection, soft cancellation, channel-dependent detection order, and randomized iteration. In the rest of this section, we discuss each in turn.

\section{A. Group Detection}

Group multiuser detection trades off complexity for performance. It breaks the users into $N_{G}$ groups of size $G$, and detects the groups in succession. During detection, all users outside of the current group are suppressed with a linear method, such as ZF or MMSE. Joint ML or joint MAP (JMAP) detection is then applied to the current group's modified measurements. Successive interference cancellation is used to remove the effects of the detected symbols of one group from the measurement before detection of the next group. This is an exponentially complex scheme like JML. However, its complexity is proportional to the $N_{G}^{\text {th }}$ root of the complexity of JML; depending on the size of the group, it can be significantly less complex than JML. The price is suboptimal detection.

To denote the formation of groups, we break b into subvectors $\mathbf{b}_{i}$, each of length $G$, and break $\mathbf{H}$ into $N_{G}$ submatrices $\mathbf{H}_{i}$, each $M \times G$, consisting of the columns associated with $\mathbf{b}_{i}$. For the desired group $j$, where all other $N_{G^{-}}$groups are therefore undesired,

$$
\mathbf{y}=\mathbf{H}_{j} \mathbf{b}_{j}+\sum_{\substack{i=0 \\ i \neq j}}^{N_{G}} \mathbf{H}_{i} \mathbf{b}_{i}+\mathbf{n}=\mathbf{H}_{j} \mathbf{b}_{j}+\mathbf{u}_{j}
$$

where $\mathbf{u}_{j}$ represents the undesired users plus noise. This technique was first characterized by Varanasi [2] and Fain and Varanasi [3]. Where [2] introduces the concept in wideband systems, [3] shows its use in a narrowband system. Both use a ZF filter for suppression of the unwanted groups, followed by JML within each group. In [3], it was shown that the GD, by varying the size of the groups, could range in performance from the simple decorrelator to the optimum JML.

$\mathrm{Ng}$ and Sousa [4] modified the GD, replacing ZF suppression with MMSE. Because MMSE leaves residual interference, they added hard interference cancellation to good effect.

In IMUD, we simply treat $\mathbf{u}_{j}$ in (2) as zero-mean Gaussian noise. It can be shown that this is equivalent to the prior MMSE suppression of [4], but with somewhat lower complexity, since we do not form an explicit filter. For group $j$, the $M \times 1$ mean vector of $\mathbf{y}$ is

$$
\begin{aligned}
\boldsymbol{\mu}_{\mathbf{y}} & =\mathrm{E}\left[\mathbf{y} \mid \mathbf{b}_{j}, \mathbf{H}\right] \\
& =\mathbf{H}_{j} \mathbf{b}_{j}
\end{aligned}
$$

and the $M \times M$ covariance matrix is

$$
\begin{aligned}
\mathbf{R}_{U} & =\mathrm{E}\left[\left(\mathbf{y}-\boldsymbol{\mu}_{\mathbf{y}}\right)\left(\mathbf{y}-\boldsymbol{\mu}_{\mathbf{y}}\right)^{\dagger} \mid \mathbf{b}_{j}, \mathbf{H}\right] \\
& =\sum_{i>j}^{N_{G}} \mathbf{H}_{i} \mathbf{H}_{i}^{\dagger}+\frac{1}{2 \Gamma} \cdot \mathbf{I}_{M}
\end{aligned}
$$

where the $U$ stands for undesired and we proceed through the groups sequentially from $j=1 \ldots N_{G}$. Assuming that $\mathbf{u}_{j}$ is Gaussian, we find the conditional probability

$$
\operatorname{Pr}\left(\mathbf{y} \mid \mathbf{b}_{j}\right)=\frac{1}{\pi^{M} \cdot\left|\mathbf{R}_{U}\right|} e^{\left.\left(\mathbf{y} \cdot \mathbf{H} \mathbf{H}_{j}\right)^{\dagger}\right)^{\dagger} \mathbf{R}_{U}^{-1}\left(\mathbf{y} \cdot \mathbf{H}_{j} \mathbf{b}_{j}\right)}
$$

IMUD actually uses JMAP calculations and a posteriori probability (APP) extraction, as described further below, instead of the ML detection and hard decisions of [4]. This allows us to incorporate both soft cancellation (Section IIIB) and iterative detection (Section IIID).

\section{B. APP Extraction and Soft Cancellation}

Golden et al. in V-BLAST [5] and Ng and Sousa [4] both use hard decisions in their interference cancellation. However, IMUD is an iterative technique, and we need soft outputs that contain useful information for the next iteration. Therefore, instead of using the statistical model (3)-(5) in a group ML detection, we use it to extract APPs, and use MAP in the last iteration. Since we have these soft outputs, we use them (as in T-BLAST [7]) in place of hard decisions in the cancellation. The soft information thus provides two purposes: to develop the iteration's APPs, and to do soft interference cancellation.

We'll denote our a priori values as $\operatorname{Pr}\left(b_{n j}\right)$, where $b_{n j}$ is the $n^{\text {th }}$ bit of the $j^{\text {th }}$ group. The measurement vector used for group detection is $\mathbf{y}^{(j)}$, as explained below. We find the APP $\operatorname{Pr}\left(b_{n j} \mid \mathbf{y}^{(j)}\right)$ by:

$$
\operatorname{Pr}\left(b_{n j} \mid \mathbf{y}^{(j)}\right)=\frac{\operatorname{Pr}\left(\mathbf{y}^{(j)}, b_{n j}\right)}{\sum_{k} \operatorname{Pr}\left(\mathbf{y}^{(j)}, b_{n j}\right)}
$$

where

$$
\operatorname{Pr}\left(\mathbf{y}^{(j)}, b_{i j}=k\right)=\sum_{\mathbf{b}_{j}=\left(b_{i j}=k\right)}\left[\operatorname{Pr}\left(\mathbf{y}^{(j)} \mid \mathbf{b}_{j}\right) \prod_{l=1}^{G} \operatorname{Pr}\left(b_{l j}\right)\right]
$$

and $\operatorname{Pr}\left(\mathbf{y}^{(j)} \mid \mathbf{b}_{j}\right)$ from (5) incorporates $\mathbf{y}^{(j)}$ and $\mathbf{R}_{U}^{(j)}$, see below. We now have the a posteriori mean $\mu_{j}$ of the BPSK data $\mathbf{b}_{j}$ with components

$$
\mu_{n j}=\operatorname{Pr}\left[b_{n j}=1 \mid \mathbf{y}^{(j)}\right]-\operatorname{Pr}\left[b_{n j}=-1 \mid \mathbf{y}^{(j)}\right]
$$

The remaining uncertainty is $\boldsymbol{\varphi}_{i}=\mathbf{b}_{i}-\boldsymbol{\mu}_{i}$, with components that we take as independent with a posteriori variance

$$
\sigma_{n j}{ }^{2}=1-\mu_{n j}{ }^{2}
$$


To perform soft cancellation after detection of group $j$, we update the received sample vector by subtracting the mean

$$
\mathbf{y}^{(j+1)}=\mathbf{y}^{(j)}-\mathbf{H}_{j} \boldsymbol{\mu}_{j}
$$

For the update of the variance, we represent the covariance matrix of subvector $\mathbf{b}_{i}$ as $\mathbf{R}_{\varphi i}=\mathrm{E}\left[\boldsymbol{\varphi}_{i} \boldsymbol{\varphi}_{i}^{\dagger}\right]$. For the detected groups,

$$
\mathbf{R}_{\varphi i}=\left(\begin{array}{ccc}
\sigma_{1, i}{ }^{2} & 0 & 0 \\
0 & \ddots & 0 \\
0 & 0 & \sigma_{G, i}{ }^{2}
\end{array}\right)
$$

For undetected groups, $\mathbf{R}_{\varphi i}$ is a $G \times G$ identity matrix. We rewrite (4) as

$$
\mathbf{R}_{U}^{(j+1)}=\sum_{\substack{i=1 \\ i \neq j+1}}^{N_{G}} \mathbf{H}_{i} \mathbf{R}_{\varphi i} \mathbf{H}_{i}^{\dagger}+\frac{1}{2 \Gamma} \cdot \mathbf{I}_{M}
$$

Note that now we need to include the past detected groups since we are using soft cancellation, as opposed to the hard decision group ML of Section III $A$. We then use the new $\mathbf{R}_{U}^{(j+1)}$ and modified sample vector $\mathbf{y}^{(j+1)}$ to calculate the APPs for the current group $j+1$.

We then use (6) as the a priori information for the next iteration. It is important to remember that before each iteration, the received sample vector must be returned to its starting value $\mathbf{y}$; otherwise the decisions will be based on the modified measurement values.

\section{Detection Order}

Vertical-BLAST (V-BLAST, [5]) demonstrated the value of detecting users in an order determined by the channel state H. We use the same idea in IMUD. Although it was presented originally with ZF suppression of undetected users, its MMSE variant is significantly better, so we will use MMSE V-BLAST for comparison with IMUD.

In IMUD, the ordering is based on the error variance instead of the SNR as in [5]. The symbol error vector is $\mathbf{e}=\mathbf{b}-\hat{\mathbf{b}}$ where the user symbol estimate $\hat{\mathbf{b}}=\mathbf{W}^{\dagger} \mathbf{y}$ and $\mathbf{W}$ is an MMSE filter. After some manipulation, the error variance for each user is found to be the diagonal entries of $E\left[\mathbf{e e}^{\dagger}\right]=\sigma_{e}^{2}$, formed as

$$
\begin{aligned}
& \boldsymbol{\sigma}_{e}{ }^{2}=\sigma_{b}{ }^{2}\left(\mathbf{I}_{N}-\left(\mathbf{H}^{(j) \dagger} \mathbf{H}^{(j)}+\frac{1}{\sigma_{b}{ }^{2}} \mathbf{I}_{N}\right)^{-1} \cdot \mathbf{H}^{(j) \dagger} \mathbf{H}^{(j)}\right) \\
& \text { where } \mathbf{H}^{(j)}=\left[\begin{array}{lll}
\mathbf{H}_{j} & \cdots & \mathbf{H}_{N_{G}}
\end{array}\right]
\end{aligned}
$$

$\sigma_{b}^{2}$ is the variance of the transmitted symbols and $j$ denotes the current group. When using EVM ordering with IMUD, we order the users with (13). The $G$ lowest variance users are selected for the first group. $\mathbf{b}_{i}$ and $\mathbf{H}$ are then reordered to match the new order. Previously detected groups are not included in the sort, as shown in (13). IMUD then continues with the JMAP from III $B$. Note that (13) does not take advantage of the soft information available. It assumes hard decision interference cancellation.

\section{Iterative Detection}

IMUD combines the group detection, soft-decision cancellation, and user ordering techniques with a basic iterative detection.

The algorithm exploits the APP soft information (6) generated during data detection. At the end of each iteration, when the APPs of all users have been calculated, those APPs become the a priori probabilities for the next iteration.

IMUD uses the EVM ordering from IIIC only for the first iteration. Prior to each subsequent iteration, the users are randomly reordered into new groups. This breaks up statistical dependencies introduced by the particular group partitioning of the previous iteration.

For the first iteration, we assume that all symbols are equiprobable; for BPSK, all a priori values are set to $1 / 2$. The steps for IMUD can now be summarized as follows:

1. Set the order of the users with (13).

2. For the first group,
a. find $\mathbf{R}_{U}^{(1)}(12)$
b. solve for the conditional probabilities in (5)
c. find the joint probabilities in (7)
d. find the soft-output APPs in (6)

3. For every successive group,
a. find $\mu_{j}$ and $\sigma_{j}$ in (8) and (9)
b. create $\mathbf{y}^{(j+1)}$ and $\mathbf{R}_{U}^{(j+1)}$ in (10) and (12)
c. continue from step $2 b$.

4. After all users are detected,

a. restart at step 2 after re-ordering of the users, or

b. output the APPs and make the hard decisions

\section{COMPUTATIONAL COMPLEXITY}

The order of complexity of the techniques described so far are shown in Table 1, where iter is the number of iterations for IMUD. 
Table 1

Computational Complexity for JML, MMSE V-BLAST, and IMUD in a fast-fading environment

\begin{tabular}{|l|l|}
\hline Technique & Complex Multiplies/Adds \\
\hline $\begin{array}{l}\text { Joint Maximum } \\
\text { Likelihood }\end{array}$ & $\sim M 2^{N+1}$ \\
\hline $\begin{array}{l}\text { MMSE V-BLAST } \\
\text { (using EVM) [5] }\end{array}$ & $\sim N^{2} M\left(M^{2}+N^{2}\right)$ \\
\hline $\begin{array}{l}\text { IMUD } \\
\text { (using EVM) } \\
\text { (III D) }\end{array}$ & $\sim\left(\begin{array}{l}\sim M^{3}\left(\sum_{k=1}^{N_{G}-1}(N-k G)\right)+ \\
N_{G} M\left(2^{G}(G+M)\right) \\
+N_{G} M N^{3}\end{array}\right.$ \\
\hline
\end{tabular}

The differences in each technique are easily recognizable. Both JML and IMUD contain terms that vary exponentially with the size of a joint ML decision, but JML depends on the total number of users, while IMUD depends on the group size, resulting in lower comnplexity for IMUD. For MMSE V-BLAST, the highest complexity components are $M \times M$ and $N \times N$ matrix inverses needed for the MMSE filter and EVM, respectively. These inverses need to be calculated for each user. IMUD also needs an $M \times M$ and $N \times N$ inverse for the GMAP and EVM; however, they only need to be computed once per group and therefore IMUD is less computationally complex. In a quasi-static channel, these inverses only need to be computed once per block, compared to once per symbol in fast-fading. V-BLAST becomes less complex in quasi-static fading since it does not make a joint ML decision.

\section{PERFORMANCE}

We will use V-BLAST as the principal comparison with IMUD, since it is a well-known reduced complexity detector. In particular, we use MMSE V-BLAST since it performs significantly better than the original ZF V-BLAST.

Simulations were performed with JML, MMSE V-BLAST, IMUD, and IMUD without IC (referred to as IMUD and IMUD No IC respectively) for 1 and 2 iterations. Throughout this section, the notation $\left(M, N_{G}, G\right)$ will be used to refer to the current system configuration - the number of antennas, groups and users per group. For all algorithms, group oriented or not, the total number of users is given by $N_{G} \times G$.

To demonstrate the effect of group size, a 12 antenna system was investigated with configurations of $(\mathbf{1 2 , 2 , 6 )},(\mathbf{1 2 , 3 , 4})$, $(12,4,3)$, and $(12,6,2)$ (Fig. 1-4). The IMUD technique with EVM sorting (denoted as IMUD(iter) EVM) was compared to the MMSE V-BLAST with EVM sorting. As a reference, the JML bound from [1] was plotted. As shown in [2], the group techniques span the gap of performance between the

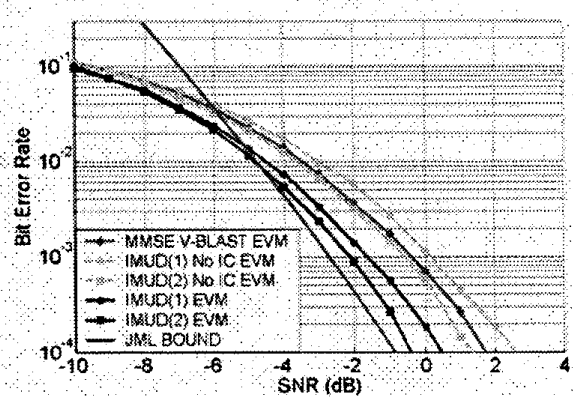

Fig. 1. $(12,2,6)$ IMUD and MMSE VBLAST Comparison

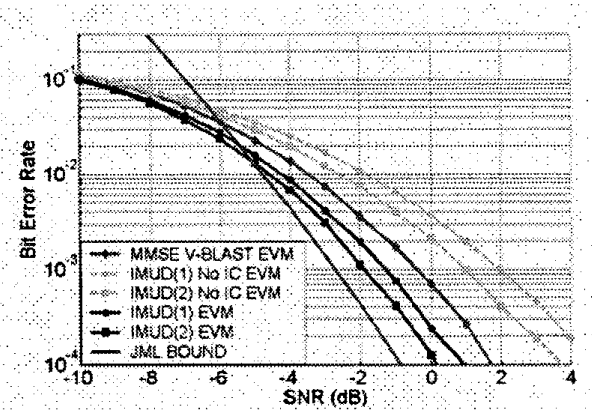

Fig. 2. $(12,3,4)$ IMUD and MMSE V-BLAST Comparison

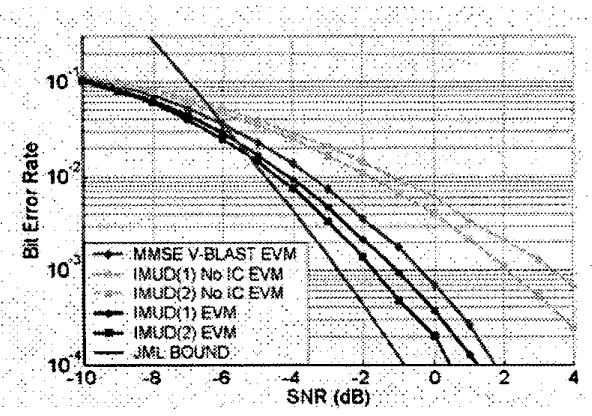

Fig. 3. $(\mathbf{1 2 , 4 , 3 )}$ IMUD and MMSE V-BLAST Comparison

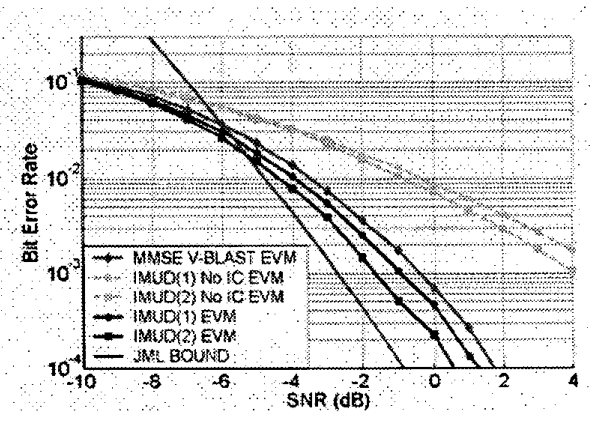

Fig. 4. (12,6,2) IMUD and MMSE V-BLAST Comparison 
linear techniques (MMSE, $(\mathbf{1 2}, \mathbf{1 2}, \mathbf{1})$ ) and the optimum (JML, $(12,1,12))$. Besides that, these graphs are instrumental in demonstrating the effects of group size on the soft-decision IC; for IMUD No IC, changes in group sizes affects the technique more than for IMUD. The soft cancellation mitigates the effects of the decrease in group size. For different group sizes, notice the SNR needed for a BER of $10^{-3}$ changes by $>4 \mathrm{~dB}$ for IMUD No IC and changes by $<1 \mathrm{~dB}$ for IMUD. Therefore we can run IMUD with smaller groups without a large drop in performance.

In the case of overloaded arrays $(N>M)$, the iterative multiuser techniques return impressive results. Fig. 5 and 6 shows the results for $(\mathbf{8 , 2 , 6 )}$ and $(\mathbf{8 , 6 , 2})$ systems. At BER of $10^{-3}$ in $(8,6,2)$, IMUD EVM performs better by $\sim 2 \frac{1}{2} \mathrm{~dB}$ than MMSE V-BLAST with only a single iteration. After the first iteration, the gain over MMSE V-BLAST is increased to $>4 \mathrm{~dB}$. With the $(8,2,6)$ system, the gain is increased to $\sim 5 \mathrm{~dB}$ for one iteration and $>7 \mathrm{~dB}$ for two iterations. There is an error floor evident in all of these techniques with overloaded arrays.

\section{CONCLUSIONS}

In this paper, a new iterative groupwise MUD technique was introduced. The structure of the MAP detection, soft cancellation and iteration were derived. Plots were generated as a comparison of existing techniques and the new iterative algorithms.

We have demonstrated that IMUD can outperform VBLAST using the same ordering technique. It seems that most of the performance gain due to iteration is achievable by the second iteration.

As a new multiuser detection technique, IMUD is quite promising. One of its biggest advantages is that it is iterative in nature, and uses soft decisions. This makes it a primary candidate for inclusion in iterative detection of multiple coded users, using IMUD in serial concatenation with the codes. There also may be applications in macrodiversity systems, where the soft decisions can be sent as $a$ priori information to other base stations within a cochannel interference area.

\section{ACKNOWLEDGEMENTS}

This work was funded by the Canada Research Chairs program and a Visiting Scholarship from Tait Electronics Ltd.

\section{REFERENCES}

[1] S. J. Grant, and J. K. Cavers, "Performance Enhancement Through Joint Detection of Cochannel Signals Using Diversity Arrays," IEEE Trans. Commun., vol. 46, pp. 1038-1049, Aug. 1998.

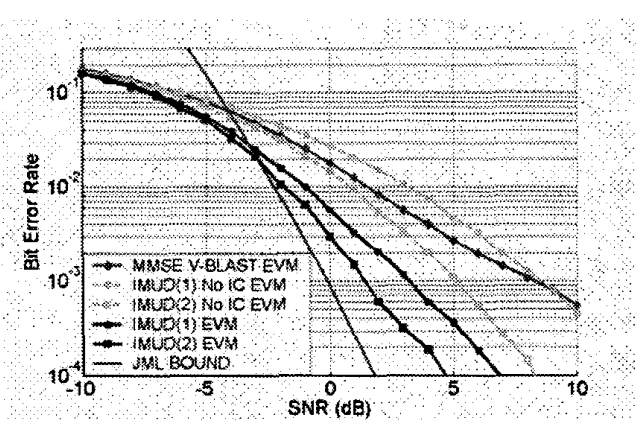

Fig. 5. Overloaded array comparison of IMUD techniques versus MMSE VBLAST in $(\mathbf{8 , 2 , 6 )}$ system

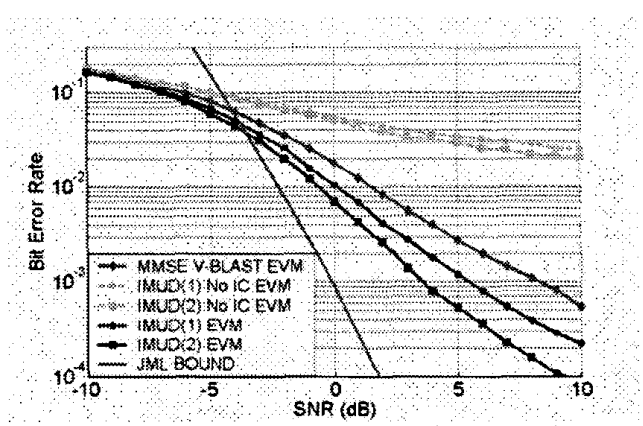

Fig. 6. Overloaded array comparison of IMUD techniques versus MMSE VBLAST in $(\mathbf{8 , 6 , 2 )}$ system.

[2] M. K. Varanasi, "Parallel Group Detection for Synchronous CDMA Communication Over FrequencySelective Rayliegh Fading Channels," IEEE Trans. Info. Theory, vol. 42, pp. 116-128, Jan. 1996.

[3] E. A. Fain, and M. K. Varanasi, "Diversity Order Gain for Narrow-Band Multiuser Communications with PreCombining Group Detection," IEEE Trans. Commun., vol. 48, pp.533-536, April 2000.

[4] B. K. Ng, and E. S. Sousa, "On Bandwidth-Efficient Multiuser-Space-Time Signal Design and Detection," IEEE J. Select. Areas Commun., vol. 20, pp. 320-329, Feb. 2002.

[5] G. D. Golden, C. J. Foschini, R. A. Valenzuela, and P. W. Wolniansky, "Detection Algorithm and Initial Laboratory Results Using V-BLAST Space-Time Communication Architecture," Electronics Letters, vol. 35, pp. 14-16, Jan. 1999.

[6] J. H. Winters, "Optimum Combining in Digital Mobile Radio with Cochannel Interference," IEEE J. Select. Areas Commun., vol. SAC-2, pp. 528-539, July 1984.

[7] M. Sellathurai, and S. Haykin, "TURBO-BLAST for High-Speed Wireless Communications," in Proc. WCNC 2000, vol. 1, pp. 315-320, Sept. 2000.

[8] John G. Proakis, Digital Communications, $4^{\text {th }}$ ed. McGraw-Hill, New York, 2000. 